\title{
Overpopulation of $\bar{\Omega}$ in $p p$ Collisions: A Way to Distinguish Statistical Hadronization from String Dynamics
}

\author{
M. Bleicher, ${ }^{1}$ F. M. Liu, ${ }^{1}$ A. Keränen, ${ }^{3}$ J. Aichelin, ${ }^{1}$ S. A. Bass, ${ }^{2}$ F. Becattini,${ }^{4}$ K. Redlich,${ }^{5,6}$ and K. Werner ${ }^{1}$ \\ ${ }^{1}$ SUBATECH, Laboratoire de Physique Subatomique et des Technologies Associées, University of Nantes-IN2P3/CNRS- \\ Ecole des Mines de Nantes, 4 rue Alfred Kastler, F-44072 Nantes Cedex 03, France \\ ${ }^{2}$ Department of Physics, Duke University, Durham, North Carolina \\ and RIKEN-BNL Research Center, Brookhaven National Laboratory, Upton, New York \\ ${ }^{3}$ Department of Theoretical Physics, University of Oulu, SF-90570 Oulu, Finland \\ ${ }^{4}$ Università di Firenze and INFN Sezione di Firenze, Via G. Sansone 1, I-50019, Sesto F.no, Florence, Italy \\ ${ }^{5}$ Theoretical Physics Division, CERN, CH-1211 Geneva 23, Switzerland \\ ${ }^{6}$ Institute for Theoretical Physics, University of Wroclaw, PL-50204 Wroclaw, Poland
}

(Received 15 November 2001; published 1 May 2002)

\begin{abstract}
The $\bar{\Omega} / \Omega$ ratio originating from string decays is predicted to be larger than unity in proton-proton interactions at SPS energies $\left(E_{\mathrm{lab}}=160 \mathrm{GeV}\right)$. The antiomega dominance increases with decreasing beam energy. This surprising behavior is caused by the combinatorics of quark-antiquark production in small and low-mass strings. Since this behavior is not found in a statistical description of hadron production in proton-proton collisions, it may serve as a key observable to probe the hadronization mechanism in such collisions.
\end{abstract}

DOI: 10.1103/PhysRevLett.88.202501

Hadron yields and their ratios stemming from the final state of ultrarelativistic heavy-ion collisions have been extensively used to explore the degree of chemical equilibrium [1-9] and to search for evidence for exotic states and phase transitions in such collisions [1]. Under the assumption of thermal and chemical equilibrium, fits with a statistical (thermal) model have been used to extract bulk properties of hot and dense matter, e.g., the temperature and chemical potential at which chemical freeze-out occurs [5-9].

The application of a statistical model to elementary hadron-hadron reactions was first proposed by Hagedorn [10] in order to describe the exponential shape of the $m_{t}$ spectra of produced particles in $p+p$ collisions. Recent analysis [11] on hadron yields in electron-positron and proton-proton interactions at several center-of-mass energies has shown that particle abundances can also be described by a statistical ensemble with maximized entropy. In fact, they are consistent with a model assuming the existence of equilibrated fireballs at a temperature $T \approx 160-170 \mathrm{MeV}$. These findings have given renewed rise to the interpretation that hadronization in elementary hadron-hadron $(h h)$ collisions is a statistical process, which is difficult to reconcile with the popular picture that hadron production in $h \mathrm{~h}$ collisions is due to the decay of color flux tubes [12], a model which has explained many dynamical features of these collisions.

In this Letter we argue that the $\bar{\Omega} / \Omega \equiv \Omega^{+} / \Omega^{-}$ratio in elementary proton-proton collisions is an unambiguous and sensitive probe to distinguish particle production via the breakup of a color flux tube from statistical hadronization.

Color flux tubes, called strings, connect two SU(3) color charges [3] and [3] with a linear confining potential. If the
PACS numbers: 25.40.Ep, 13.85.Ni, 24.10.Lx, 25.75.Dw

excitation energy of the string is high enough, it is allowed to decay via the Schwinger mechanism [13]; i.e., the rate of newly produced quarks is given by

$$
\frac{d N_{\kappa}}{d p_{\perp}} \sim \exp \left[-\pi m_{\perp}^{2} / \kappa\right]
$$

where $\kappa$ is the string tension and $m_{\perp}=\sqrt{p_{\perp}^{2}+m^{2}}$ is the transverse mass of the produced quark with mass $m$.

However, specific string models may differ in their philosophy and the types of strings that are created: (i) In URQMD [14] the projectile and target protons become excited objects due to the momentum transfer in the interaction. The resulting strings, with at most two strings being formed, are of the diquark-quark type. (ii) In NEXUS [15], the $p p$ interaction is described in terms of Pomeron exchanges or ladder diagrams. Both hard and soft interactions take place in parallel. Energy is shared equally between all cut Pomerons and the remnants. The end points of the cut Pomerons (i.e., the end points of the strings) may be valence quarks, sea quarks, or antiquarks. (iii) In PYTHIA [16], a scheme similar to that in URQMD is employed. However, hard interactions may create additional strings from scattered gluons and sea quarks. Most strings are also of diquark-quark form.

Figure 1 (left) depicts the antibaryon to baryon ratio at midrapidity in proton-proton interactions at $160 \mathrm{GeV}$. The results of the calculations by NEXUS, URQMD, and PYTHIA, which are well established string-fragmentation models for elementary hadron-hadron interactions, are included in this figure. In all these models, the $\bar{B} / B$ ratio increases strongly with the strangeness content of the baryon. For strangeness $|s|=3$ the ratio significantly exceeds unity. In URQMD and PYTHIA the hadronization of the diquark-quark strings 


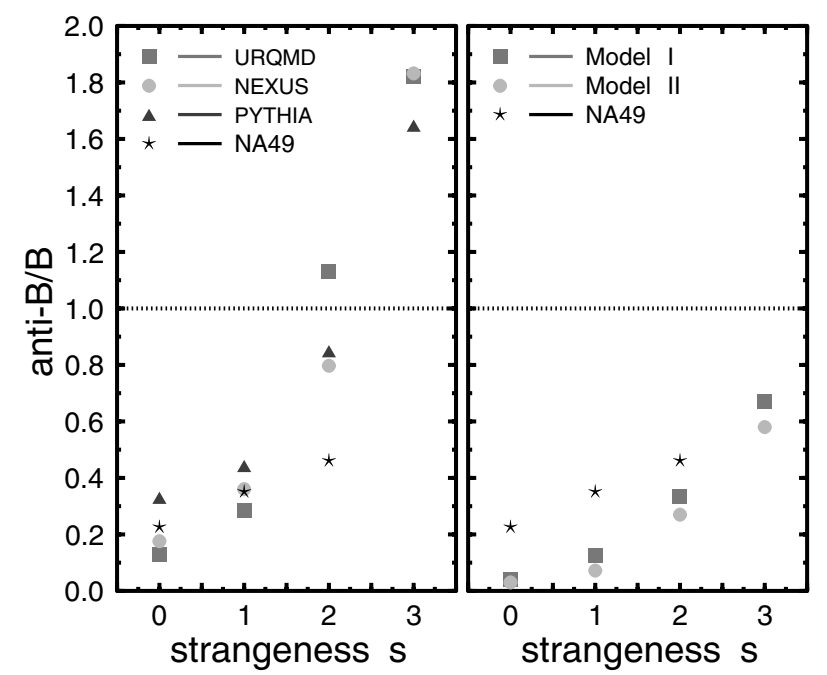

FIG. 1. Left: antibaryon to baryon ratio at $\left|y-y_{\mathrm{cm}}\right|<1$ in $p p$ interactions at $160 \mathrm{GeV}$ as given by PYTHIA, NEXUS, and URQMD. Right: antibaryon to baryon ratio for the same reaction as given by statistical models. Stars depict preliminary NA49 data for the $\bar{B} / B$ ratio at midrapidity.

leads directly to the overpopulation of $\bar{\Omega}$. In NEXUS, however, the imbalance of quarks and antiquarks in the initial state leads to the formation of $q_{\mathrm{val}}-\bar{s}_{\text {sea }}$ strings (the $s_{\text {val }}-\bar{q}_{\text {sea }}$ string is not possible). These strings then result in the overpopulation of $\bar{\Omega}$ 's.

In Fig. 1 the string model results are compared with the predictions of statistical models (SM). Within the SM, hadron production is commonly described using the grand canonical (GC) partition function, where the charge conservation is controlled by the related chemical potential. In this description a net value of a given $\mathrm{U}(1)$ charge is conserved on average. However, in the limit of small particle multiplicities, conservation laws must be implemented exactly. This is done by using the canonical (C) ensemble $[11,17,18]$. The conservation of quantum numbers in the canonical approach severely reduces the phase space available for particle production. Thus, exact charge conservation is of crucial importance in the description of particle yields in proton-induced processes and in $e^{+} e^{-}$[11], as well as in peripheral heavy-ion collisions [18].

In Fig. 1 the predictions of two different statistical models for $\bar{B} / B$ ratios in $p p$ collisions are included. The main difference between these models is the implementation of baryon number and electric charge conservation and the way an additional strangeness suppression is introduced.

(I) The calculation in this statistical model [19] is a full canonical one with fixed baryon number, strangeness, and electric charge identical to those of the initial state. Also, an extra strangeness suppression is introduced to reproduce the experimental multiplicities. This is done by considering the number of newly produced $\langle s \bar{s}\rangle$ pairs as an additional charge to be found in the final hadrons. The $s \bar{s}$ pairs fluctuate according to a Poisson distribution and its mean number is considered as a free parameter to be fitted [19]. The parameters used for the prediction of the $\Omega^{+} / \Omega^{-}$ratio $(T$, global volume $V$ sum of single cluster volumes and $\langle s \bar{s}\rangle)$ have been obtained by a fit to preliminary NA49 $p p$ data [20] yielding $T=183.7 \pm 6.7 \mathrm{MeV}$, $V T^{3}=6.49 \pm 1.33$, and $\langle s \bar{s}\rangle=0.405 \pm 0.026$ with a $\chi^{2} /$ d.o.f. $=11.7 / 9$. It must be pointed out that the $\Omega^{+} / \Omega^{-}$ratio is actually independent of the $\langle s \bar{s}\rangle$ parameter and depends only on $T$ and $V$ (see also Fig. 4).

(II) Here the conservation of baryon number and electric charge is approximated by using the GC ensemble. Under thermal conditions at top SPS energy this approximation leads to deviations from the exact $\mathrm{C}$ results in $p p$ collisions by at most $20 \%-30 \%$ [21]. Strangeness conservation is, however, implemented on the canonical level following the procedure proposed in [18]. It accounts for strong correlations of produced strange particles due to constraints imposed by the locality of the conservation laws. In $p p$ collisions strangeness is assumed not to be distributed in the whole volume of the fireball but to be locally strongly correlated. A correlation volume parameter $V_{0}=4 \pi R_{0}^{3} / 3$ is introduced, where $R_{0} \sim 1 \mathrm{fm}$ is a typical scale of QCD interactions. The previous analysis of WA97 $p A$ data yields $R_{0} \sim 1.12 \mathrm{fm}$ corresponding to $V_{0} \simeq 6 \mathrm{fm}^{3}$. Note that, however, hidden strange particles are not canonically suppressed in this approach. The analysis of experimental data in AA collisions has shown that $T$ and $\mu_{B}$ are almost entirely determined by the collision energy and depend only weakly on the number of participants [6]. The $4 \pi$ results of NA49 on $\bar{p} / \pi$ and $\pi / A_{\text {part }}$ ratios in $p-p$ and $\mathrm{Pb}-\mathrm{Pb}$ collisions coincide within $20 \%-30 \%$. In terms of the SM this can be understood if $T$ and $\mu_{B}$ in $p-p$ and $\mathrm{Pb}-\mathrm{Pb}$ collisions have similar values. We take $T \simeq 158 \mathrm{MeV}$ and $\mu_{B} \simeq 238 \mathrm{MeV}$ as obtained from the SM analysis of full phase-space NA49 $\mathrm{Pb}-\mathrm{Pb}$ data [6]. The volume of the fireball $V \sim 17 \mathrm{fm}^{3}$ and the charge chemical potential in $p p$ was then found to reproduce the average charge and baryon number in the initial state.

The predictions of the statistical models are shown in Fig. 1 (right). In these approaches the $\bar{B} / B$ ratio is seen to exhibit a significantly weaker increase with the strangeness content of the baryon than expected in the string fragmentation models. For comparison, both figures include preliminary data on the $\bar{B} / B$ ratios obtained at midrapidity by the NA49 Collaboration [20].

The predictions of the statistical models in Fig. 1 refer to full phase-space particle yields while measurements of $\bar{B} / B$ ratios in $p p$ collisions have been performed at midrapidity, where they are expected to be the largest. Therefore, sizable deviations of the model results from the data seen in Fig. 1 are to be expected. The problem of rapidity and transverse momentum distributions in the statistical model has been discussed in Refs. [19,22]. However, we emphasize that the key result $\bar{B} / B<1$ is not expected to change.

The rapidity dependence of the $\Omega$ and $\bar{\Omega}$ yield is presented in Fig. 2 within different string models. The results were calculated in $p p$ interactions at $160 \mathrm{GeV}$ within 


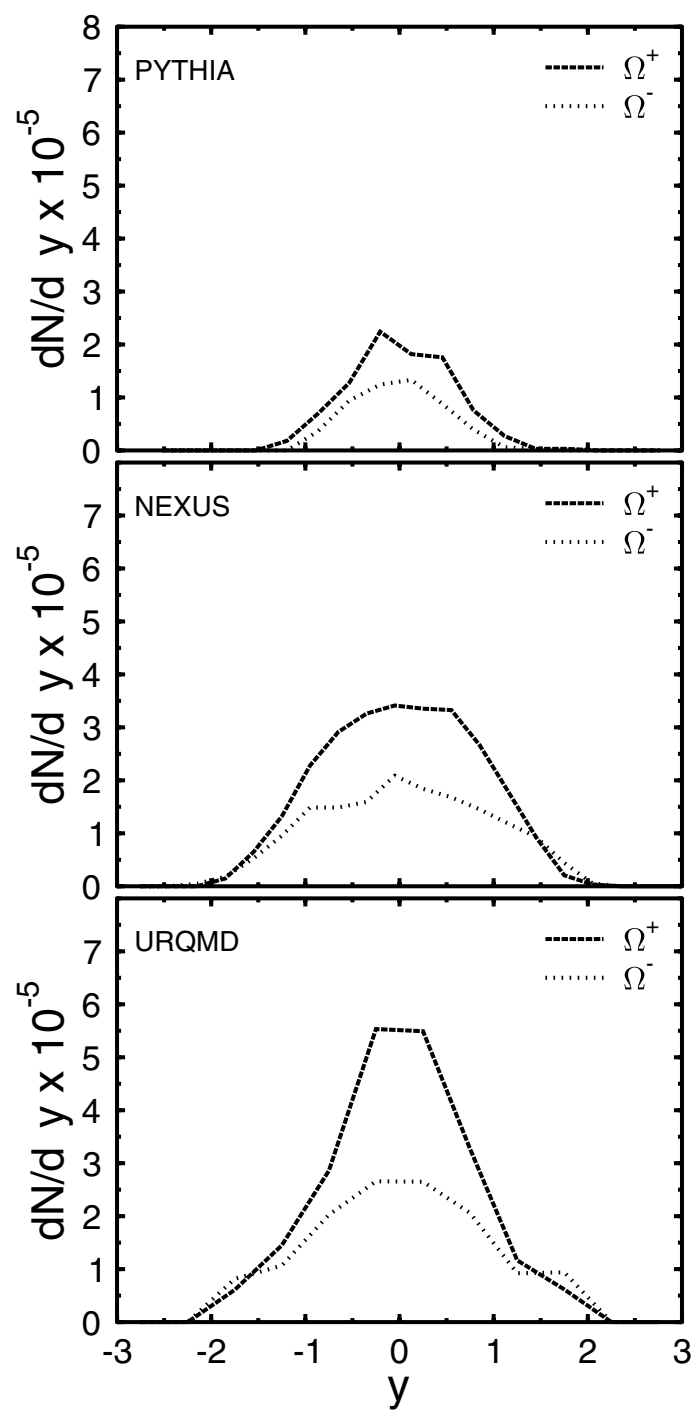

FIG. 2. Rapidity density of $\bar{\Omega}$ and $\Omega$ in $p p$ interactions at $160 \mathrm{GeV}$ as predicted by URQMD, NEXUS, and PYTHIA.

PYTHIA, NEXUS, and URQMD (from top to bottom). As can be seen, the $\bar{\Omega} / \Omega$ ratio is largest around midrapidity.

The $\bar{\Omega} / \Omega$ ratio is fairly robust — different string-model implementations (PYTHIA, URQMD, NEXUS) all agree in their predictions within $\pm 20 \%$. However, as shown in Table I the total $4 \pi$ yields of $\Omega$ 's and $\bar{\Omega}$ 's may differ by a factor of 4 between the different string models. The statistical models give in general more consistent results; however, deviations up to $30 \%$ are not excluded. In string models, the particle abundances depend on the parameters

TABLE I. Predictions of different models on the $4 \pi$ yield of $\Omega$ and $\bar{\Omega}$ in $p p$ collisions at $160 \mathrm{GeV}$.

\begin{tabular}{lcc}
\hline \hline \multicolumn{1}{c}{ Model } & $\Omega\left(\times 10^{-4}\right)$ & $\bar{\Omega}\left(\times 10^{-4}\right)$ \\
\hline NEXUS & 0.48 & 0.79 \\
PYTHIA & 0.17 & 0.30 \\
URQMD & 0.66 & 1.05 \\
Canonical model I & 0.46 & 0.31 \\
Canonical model II & 0.41 & 0.24 \\
\hline \hline
\end{tabular}

chosen for the fragmentation scheme, while in statistical models they reflect the differences between the ensembles chosen. Thus, the absolute yields allow a distinction to be made between the implementations once experimental data become available. We show now that this is a generic feature that string models give $\bar{\Omega} / \Omega>1$, whereas statistical models yield $\bar{\Omega} / \Omega<1$ in $p p$ interactions.

In order to understand the large $\bar{\Omega} / \Omega$ values predicted by string models one elucidates in Fig. 3 the color flux tube breakup mechanism. Figure 3 shows the fragmentation of the color field into quark-antiquark pairs, which then coalesce into hadrons. While in large strings $\Omega$ 's and $\bar{\Omega}$ 's are produced in equal abundance (a), low-mass strings in URQMD suppress $\Omega$ production at the string ends (b), while in NEXUS $\bar{\Omega}$ 's are enhanced (c). Thus, the microscopic method of hadronization leads to a strong imbalance in the $\bar{\Omega} / \Omega$ ratio in low-mass strings.

The $\bar{\Omega} / \Omega$ ratio depends in a strongly nonlinear fashion on the mass of the fragmenting string. Figure 4 shows the $\bar{\Omega} / \Omega$ ratio as a function of the mass of the fragmenting string (i.e., different beam energies in $p p$ ). One clearly observes a strong enhancement of $\bar{\Omega}$ production at low energies, while for large string masses the ratio approaches the value of $\bar{\Omega} / \Omega=1$ (which should be reached in the limit of an infinitely long color flux tube).

Statistical models, on the other hand, are not able to yield a ratio of $\bar{\Omega} / \Omega>1$. This can be easily understood in the $\mathrm{GC}$ formalism, where the $\bar{B} / B$ ratio is very sensitive to the baryon chemical potential $\mu_{B}$. For finite baryon densities, the $\bar{B} / B$ ratio will always be $<1$ and only in the limit of $\mu_{B}=0$ may $\bar{\Omega} / \Omega=1$ be approached. These features survive in the canonical framework, where the GC fugacities are replaced by ratios of partition functions $[11,23]$. This is shown in Fig. 4 (right) where the ratio $\bar{\Omega} / \Omega$ in $p p$ collisions (according to the previously described model I) is plotted as a function of volume for four different temperatures. Hence, finite size corrections in the statistical model actually lead to the opposite behavior [24] in the

a)

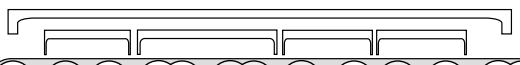

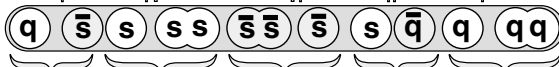
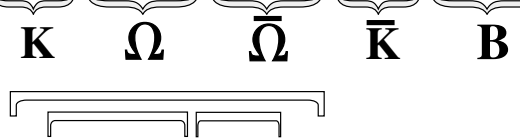

b)

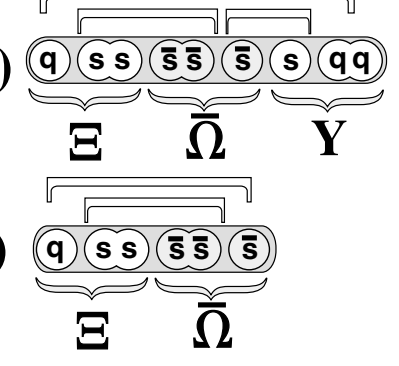

FIG. 3. Fragmentation of a color field into quarks and hadrons. While in large strings $\Omega$ 's and $\bar{\Omega}$ 's are produced in equal abundance (a), small diquark strings suppress $\Omega$ 's at the string ends (b), and sea- $\bar{s}$ quarks enhance $\bar{\Omega}$ 's (c). 


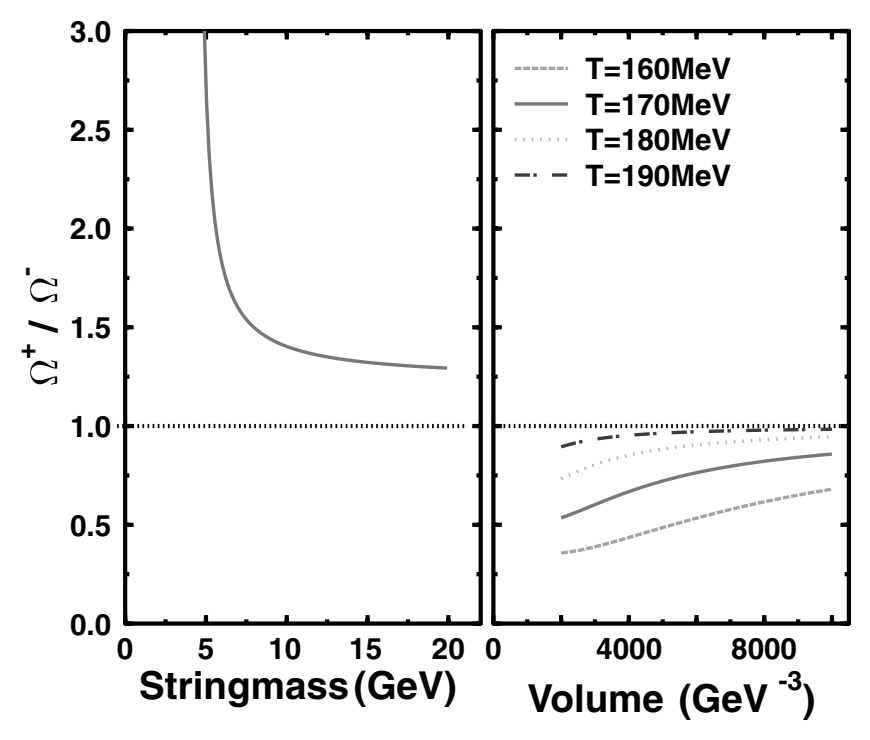

FIG. 4. Left: $\bar{\Omega} / \Omega$ ratio as a function of string mass. Right: $\bar{\Omega} / \Omega$ ratio as a function of the volume in model $\mathrm{I}$.

ratio of $\bar{\Omega} / \Omega$ vs system size (i.e., volume replacing string mass) to that observed in the fragmenting color flux tube picture.

In conclusion, within the fragmenting color flux tube models we have predicted that the $\bar{\Omega} / \Omega$ ratio is significantly above unity. This is in strong contrast to statistical model results, which always imply that $\bar{B} / B$ ratios are below or equal to unity in proton-proton reactions. Since this observable is accessible to NA49 measurements at the SPS it can provide an excellent test to distinguish the statistical model hadronization scenario from that of microscopic color flux tube dynamics.

We thank K. Kadija (NA49 Collaboration) for fruitful and stimulating discussions. S. B. acknowledges support from RIKEN, Brookhaven National Laboratory, and DOE Grants No. DE-FG02-96ER40945, as well as No. DEAC02-98CH10886. K. R. acknowledges Polish Committee for Scientific Research Grant No. KBN-2P03B-03018. M. B. acknowledges support from the region Pays de la Loire.

[1] S. A. Bass, M. Gyulassy, H. Stöcker, and W. Greiner, J. Phys. G 25, R1 (1999); H. Satz, Rep. Prog. Phys. 63, 1511 (2000).

[2] H. Stöcker, W. Greiner, and W. Scheid, Z. Phys. A 286, 121 (1978); D. Hahn and H. Stöcker, Nucl. Phys. A452, 723 (1986).

[3] R. Stock, Phys. Rep. 135, 261 (1986); Phys. Lett. B 456, 277 (1999).
[4] J. Rafelski and B. Müller, Phys. Rev. Lett. 48, 1066 (1982); J. Rafelski, Phys. Rep. 88, 331 (1982); P. Koch, B. Müller, and J. Rafelski, Phys. Rep. 142, 167 (1986).

[5] J. Letessier, A. Tounsi, U. Heinz, J. Sollfrank, and J. Rafelski, Phys. Rev. Lett. 70, 3530 (1993); J. Letessier, J. Rafelski, and A. Tounsi, Phys. Lett. B 321, 394 (1994); J. Rafelski and M. Danos, Phys. Rev. C 50, 1684 (1994).

[6] J. Cleymans and K. Redlich, Phys. Rev. Lett. 81, 5284 (1998); F. Becattini, J. Cleymans, A. Keranen, E. Suhonen, and K. Redlich, Phys. Rev. C 64, 024901 (2001).

[7] P. Braun-Munzinger, I. Heppe, and J. Stachel, Phys. Lett. B 465, 15 (1999).

[8] P. Braun-Munzinger, J. Stachel, J. P. Wessels, and N. Xu, Phys. Lett. B 344, 43 (1995); 365, 1 (1996); P. BraunMunzinger and J. Stachel, Nucl. Phys. A606, 320 (1996); P. Braun-Munzinger, D. Magestro, K. Redlich, and J. Stachel, Phys. Lett. B 518, 41 (2001).

[9] C. Spieles, H. Stocker, and C. Greiner, Eur. Phys. J. C 2, 351 (1998); C. Greiner and H. Stöcker, Phys. Rev. D 44, 3517 (1991); S. A. Bass et al., Phys. Rev. Lett. 81, 4092 (1998).

[10] R. Hagedorn, Nuovo Cimento Suppl. 3, 147 (1965); R. Hagedorn and J. Ranft, Nuovo Cimento Suppl. 6, 169 (1968).

[11] F. Becattini, Z. Phys. C 69, 485 (1996); F. Becattini and U. Heinz, Z. Phys. C 76, 269 (1997).

[12] A. Casher, H. Neuberger, and S. Nussinov, Phys. Rev. D 20, 179 (1979).

[13] J. S. Schwinger, Phys. Rev. 82, 664 (1951).

[14] M. Bleicher et al., J. Phys. G 25, 1859 (1999); S. A. Bass et al., Prog. Part. Nucl. Phys. 41, 225 (1998).

[15] H. J. Drescher et al., Phys. Rep. 350, 93 (2001).

[16] H.-U. Bengtsson and T. Sjöstrand, Comput. Phys. Commun. 46, 43 (1987).

[17] R. Hagedorn, CERN Report No. 71, 1971; E. Shuryak, Phys. Lett. 42B, 357 (1972); J. Rafelski, Phys. Lett. 97B, 279 (1980); K. Redlich and L. Turko, Z. Phys. C 5, 201 (1980); C. M. Ko, V. Koch, Z. Lin, K. Redlich, M. Stephanov, and X. N. Wang, Phys. Rev. Lett. 86, 5438 (2001); P. Braun-Munzinger, J. Cleymans, H. Oeschler, and K. Redlich, hep-ph/0106066.

[18] J. S. Hamieh, K. Redlich, and A. Tounsi, Phys. Lett. B 486, 61 (2000); J. Phys. G 27, 413 (2001).

[19] F. Becattini and G. Passaleva, hep-ph/0110312 [Eur. Phys. J. (to be published)].

[20] J. Bachler et al., Nucl. Phys. A661, 45 (1999); R. A. Barton et al., J. Phys. G 27, 367 (2001); V. Afanasev et al., Phys. Lett. B 491, 59 (2000); M. Gazdzicki (private communication).

[21] M. Gorenstein and M. Gazdzicki, Phys. Lett. B 483, 60 (2000).

[22] F. Becattini, M. Gazdzicki, and J. Sollfrank, Eur. Phys. J. C 5, 143 (1998).

[23] R. Hagedorn and K. Redlich, Z. Phys. C 27, 541 (1985); J. Cleymans, K. Redlich, and E. Suhonen, Z. Phys. C 51, 137 (1991).

[24] F. Becattini and A. Keränen (to be published). 